\title{
Probelab ReImager: A Multi-Platform, Open Source Software for Electron Image and X-ray Map Visualization and Customization
}

\author{
Mia Kraft and Anette von der Handt
}

Department of Earth and Environmental Sciences, University of Minnesota, Minneapolis, Minnesota, United States

High-resolution electron images and X-ray maps are routinely produced in electron microscopy and microanalysis laboratories. For publication and documentation purposes, microscopists require access to the best available resolution of the images and often desires further annotation options.

While vendor software excels in data acquisition, it often limits image annotation options and lacks exportation in modern file formats. Even more, a no-cost, easy-to-use software that runs on multiple operating systems and has low demands on hardware requirements is especially valuable during the prevalence of remote work during the COVID pandemic and the associated lack of access to lab-grade computer and software infrastructure.

Our lab uses the Thermo Scientific Pathfinder (and its predecessor NSS) software to capture electron images and X-ray maps on our JEOL JXA8530FPlus electron microprobe. This software offers data export to various report templates but lacks batch export options for images in their native resolution and only allows very limited visual customization. To fill this gap, we have created the program "Probelab ReImager", which facilitates easy batch exportation and customization - including annotations - of electron images and X-ray intensity maps.

Pathfinder/NSS allows acquisition of spectra as points, rectangles, circles, or polygons with documentation of their relative location on an electron image. Probelab ReImager supports visualization of these acquisition types, expands on their customization options and allows quick processing and then batch exporting. Current annotation features include: customizable scale bar location and appearance; spectral data positions can be displayed, hidden or colored for optimal contrast individually; form and size of position markers can be customized (Figure 1). For point data, the user can choose between four different colors and four different marker types and their relative size can be user-defined as a percentage of the image size.

Pathfinder/NSS offers hyperspectral imaging, and an impressive library of X-ray map processing and filtering features is available. The software gives control to what elements to display and color scales but forces a layer order and all layers have no opacity. Probelab ReImager provides further customization of layer opacity, layer order and the choice of display on either the electron image or a solid background. By introducing layer opacity as a variable, it gives the further benefit to minimize noise in low intensity maps as opacity mixes multiple colors on the same pixel. In the Pathfinder output, any noise in the top layer map visually dominates the final map. This is shown in Figure 2.

Image and X-ray map customization can be uniquely stored and exported, even allowing multiple versions for each exported image if desired. While many programs still only export images as TIFFs, this archaic image format has been superseded by formats such as PNG for lossless and JPG for lossy compression. Both of these formats offer more advanced metadata and compression, and are supported on every operating system. To facilitate the change of image formats, Probelab ReImager by default exports images to PNG but also supports JPG, WebP, and TIFF.

Probelab ReImager is written in NodeJS utilizing Electron, resulting in a mobile and flexible web browserbased desktop application. It currently supports Windows 7, 8, and 10, and MacOS (back to El Capitan). Threading of image processing allows for faster processing of images without lag or "freezing" during use. 
Image processing can be intense, especially for 4096-resolution images, thus an average of $300 \mathrm{MB}$ of memory is required while running. On an average Intel i5 5th generation CPU, it fully processes, customizes, and exports a 4096-resolution image in less than 20 seconds, with mobile processors taking up to 30 seconds. Multiple layers of cache are used to increase image processing and rendering. The image displayed to the user is a PNG generated by the backend process. This image is cached as layers, which allow for quick change of $\mathrm{X}$-ray map layers, individual points, and scale bar position without re-rendering large portions of the image.

In addition to Thermo Scientific's Pathfinder/NSS software, Probelab ReImager supports scale bar customization for electron images acquired with JEOL EPMA and SEM-platform software. Probelab ReImager is released to the public and available at https://reimager.probelab.net. Code is available on Github through the webpage and licensed under MIT copyright license.

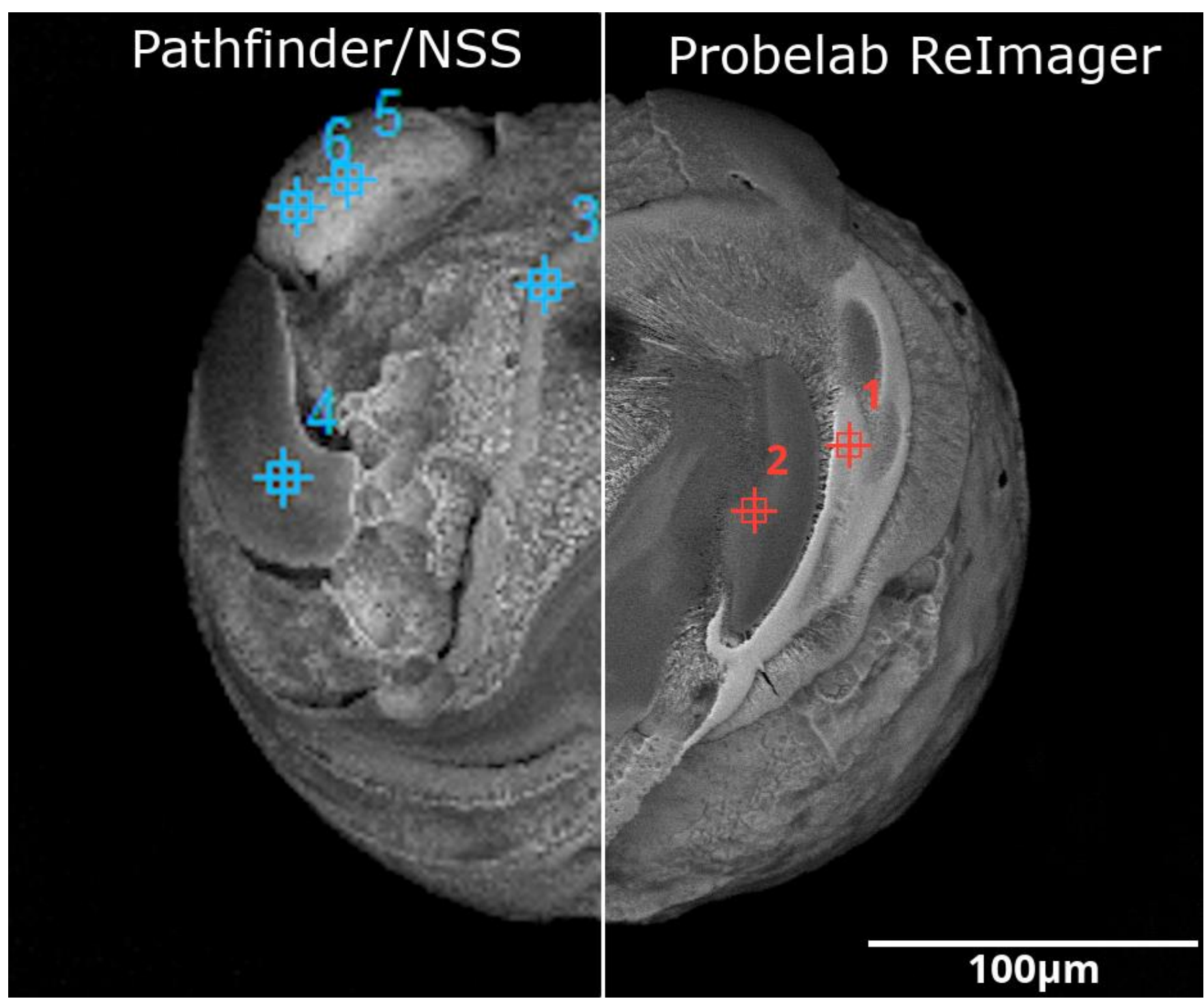

Figure 1. Comparison of the same image exported through the Pathfinder/ NSS software (left) and through Probelab ReImager (right). The left image export is more pixelated because the software down-samples exported images relative to their native resolution. Also, the interface does not provide any customization of location markers as shown for the spectra acquisitions. Probelab ReImager exports in native resolution without down-sampling. 


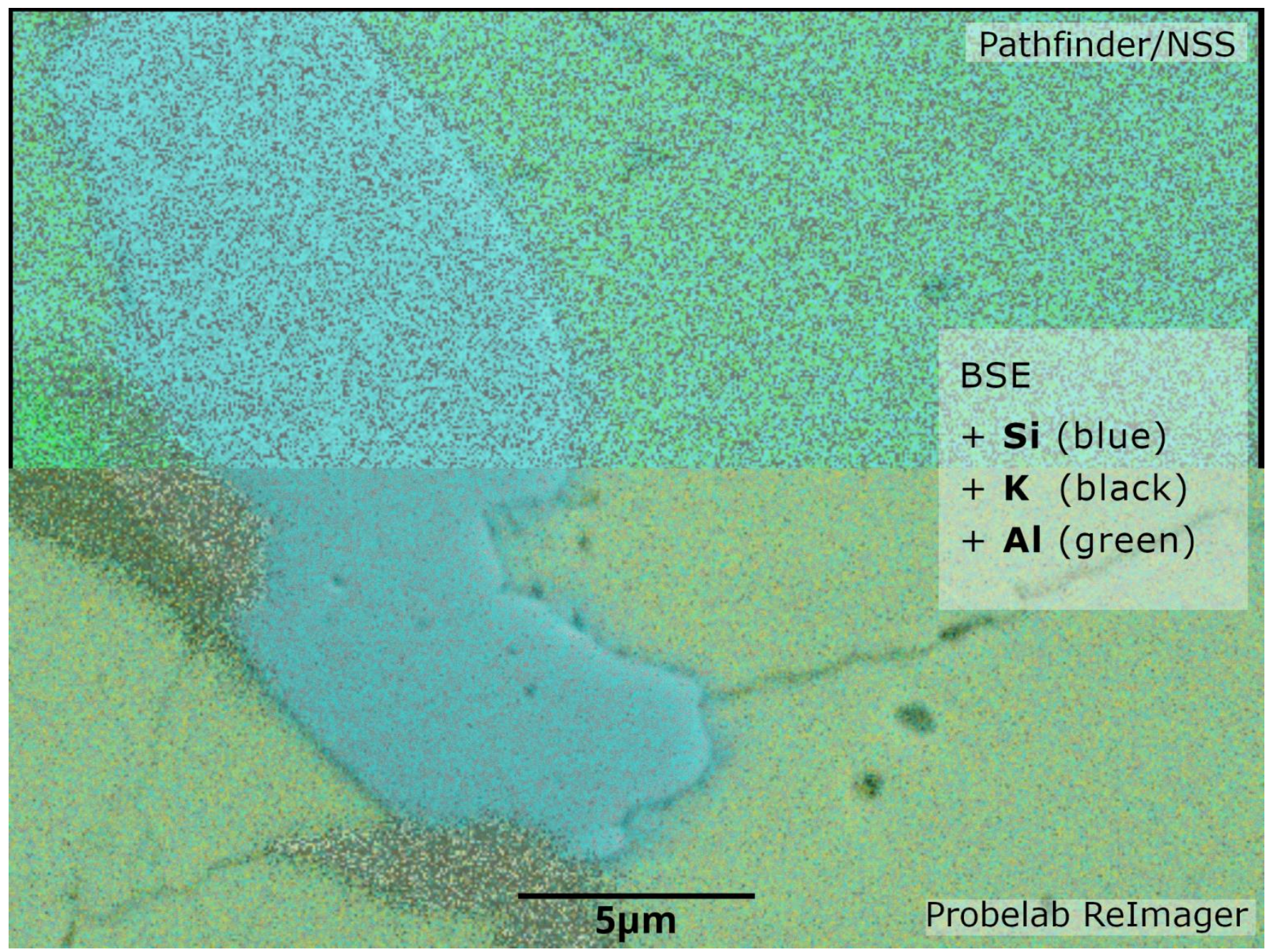

Figure 2. Comparison between outputs from Pathfinder/NSS (top) and Probelab ReImager (bottom). In both cases, X-ray maps were processed previously in Pathfinder with the Hot Pixel Suppression filter and manual contrast of K. Probelab ReImager's opacity options (set at 50\% in this example) improve the noise of the raw map output and image clarity. In addition, output from Pathfinder adds a thin black edge around the maps.

\section{References}

[1] Kraft, N. \& von der Handt, A. (2021): Probelab ReImager: An Open-Source Software for Streamlining Image Processing in an Electron Microscopy Laboratory. Microscopy Today. doi:10.1017/S1551929521000481

[2] Support for this research came from the National Science Foundation: EAR-1849465 (AVDH). 